\title{
Understanding the climate of the past 2000 years: Phase 3 of the PAGES $2 \mathrm{k}$ Network
}

\author{
PAGES 2k Network Coordinators*
}

Zaragoza, Spain, 11 May 2017

\begin{abstract}
The PAGES $2 k$ Network was created with the aim of reconstructing changes in regional and global surface climate over the past 2000 years. During Phases 1 (2008-2013) and 2 (2014-2016), regional and trans-regional groupings focused on building reconstructions for terrestrial regions and the oceans. The conclusion of Phase 2 coincided with the release of an open and transparent global database of temperature-sensitive proxies spanning the Common Era (Fig. 1; PAGES $2 k$ Consortium 2017). This product will be followed up by a special issue of Climate of the Past, entitled "Climate of the past 2000 years: regional and trans-regional syntheses" (www.clim-past.net/special_issue841.html).
\end{abstract}

The third phase of the PAGES $2 \mathrm{k}$ Network was launched in May 2017 at the PAGES 5th Open Science Meeting (OSM). In this new phase, we aim to address major questions centered on past hydroclimate, climate processes and proxy uncertainties, with three distinct scientific themes:

- Theme 1 (Climate Variability, Modes and Mechanisms) aims to further understand the mechanisms driving regional climate variability and change on interannual to centennial time scales.
- Theme 2 (Methods and Uncertainties) aims to reduce uncertainties in the interpretation of observations imprinted in paleoclimatic archives by environmental sensors.

\section{- Theme 3 (Proxy and Model} Understanding) aims to identify and analyze the extent of agreement between reconstructions and climate model simulations.

At the OSM, anyone interested in the network was invited to a lunchtime meeting. The goals of this meeting were to present the framework for Phase 3, introduce the new coordination team, present proposals for new projects, and collect ideas for additional Phase 3 activities.

To stimulate new ideas for projects, a "Post-It" note exercise was held, where participants were asked to nominate scientific questions they would like PAGES $2 k$ to address. This exercise led us to identify five themes that sparked widespread interest, and which could serve as foci for potential Phase 3 projects: (i) the combination of highand low-resolution proxies; (ii) data stewardship and script availability; (iii) data-model comparison; (iv) the evolution of the mid- to

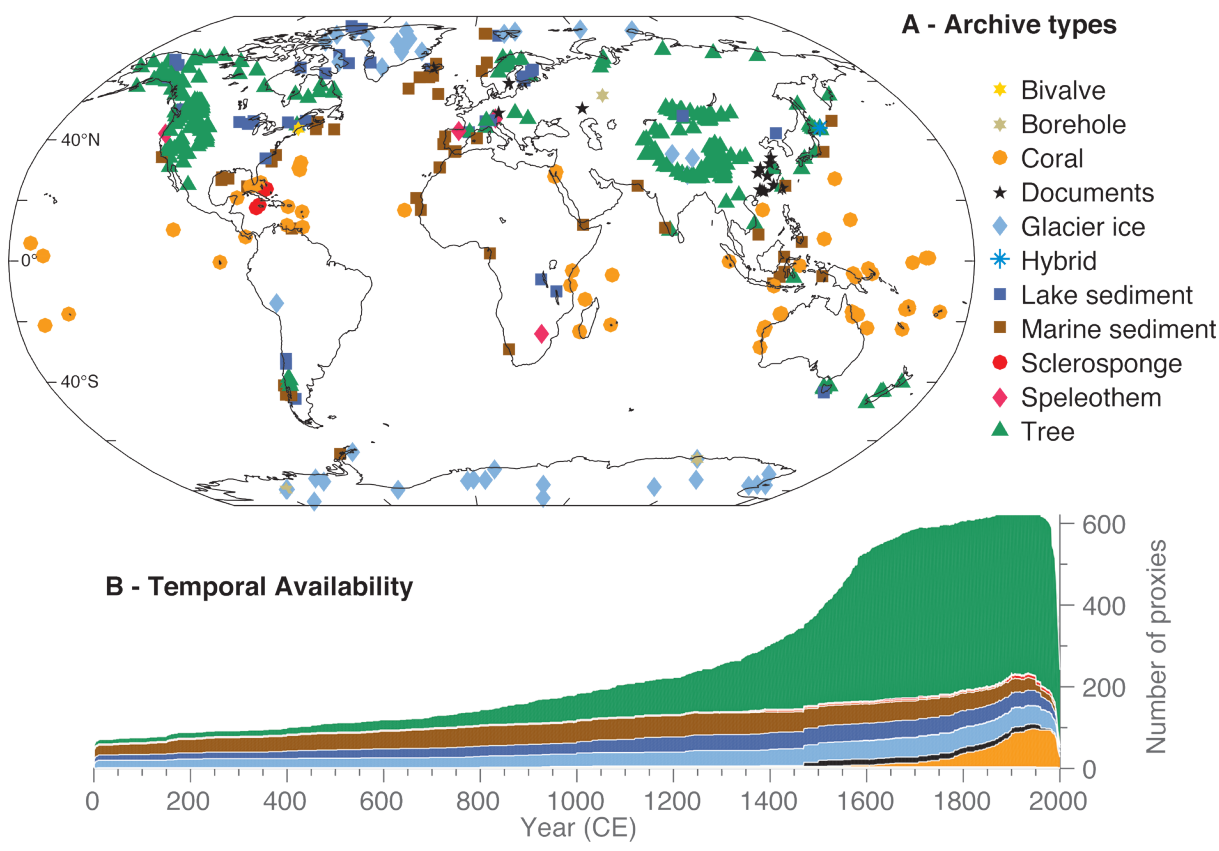

Figure 1: Location and length of the 692 records in the new PAGES $2 \mathrm{k}$ global temperature database v2.0.0. This resource will serve as a basis for many $2 \mathrm{k}$ projects. (A) Geographical distribution, by archive type, and (B) temporal availability. Modified from PAGES 2k Consortium (2017). high-latitude Southern Hemisphere climate; and (v) the hydrological cycle, temperaturehydroclimate co-variability and interannual to interdecadal variability. Participants split into groups to discuss potential projects in each of these fields.

The overall structure of PAGES $2 k$ consists of a network of projects, each organized and led by members of the community. So far, nine projects have begun their activities. These are either trans-regional projects that have transitioned from Phase 2, or new projects that have been proposed and endorsed as part of Phase 3. Examples of Phase 3 projects include: Clivash2k, which will generate a community-based multi-proxy reconstruction of key modes of Southern Hemisphere variability over the past 2000 years; and Iso2k, which started its activities during Phase 2 and aims to create a global database of paleo- $\delta^{18} \mathrm{O}$ and $\delta \mathrm{D}$ records. New projects can be proposed at any time.

Phase 3 of the PAGES $2 k$ Network will run until 2020 and is open to anyone who is interested. There are a number of ways to participate: by contributing your data or expertise, by initiating a new project or by participating in an existing one. If you would like to participate in PAGES 2k, please contact us. To receive updates on our activities, sign up for the mailing list at https://listserv. unibe.ch/mailman/listinfo/pages2k.pages

For further information on the PAGES 2k Network, including details of existing projects and the procedure for proposing a new one, please see www.pastglobalchanges.org/ ini/wg/2k-network

\section{AFFILIATIONS}

N. Abram (Australian National University, Australia), O. Bothe (Helmholtz-Zentrum Geesthacht, Germany), H. Linderholm (Gothenburg University, Sweden), B Martrat (Spanish Council for Scientific Research, Spain), H. McGregor (University of Wollongong, Australia), R. Neukom (University of Bern, Switzerland), S. Phipps (University of Tasmania, Australia), S. St George (University of Minnesota, USA), L. von Gunten (PAGES IPO, Switzerland).

\section{CONTACT}

Lucien von Gunten: lucien.vongunten@pages.unibe.ch REFERENCES

PAGES 2k Consortium (2017) Sci Data 4:170088 